\title{
High-Frequency RCS of Complex Radar Targets in Real-Time
}

\author{
Juan M. Rius, Member, IEEE, Miguel Ferrando, Member, IEEE, and Luis Jofre, Member, IEEE
}

\begin{abstract}
This paper presents a new and original approach for computing the high-frequency radar cross section (RCS) of complex radar targets in real time with a 3-D graphics workstation. The aircraft is modeled with I-DEAS solid modeling software using a parametric surface approach. High-frequency RCS is obtained through physical optics (PO), method of equivalent currents (MEC), physical theory of diffraction (PTD), and impedance boundary condition (IBC). This method is based on a new and original implementation of high-frequency techniques which we have called graphical electromagnetic computing (GRECO). A graphical processing approach of an image of the target at the workstation screen is used to identify the surfaces of the target visible from the radar viewpoint and obtain the unit normal at each point. High-frequency approximations to RCS prediction are then easily computed from the knowledge of the unit normal at the illuminated surfaces of the target. The image of the target at the workstation screen (to be processed by GRECO) can be potentially obtained in real time from the I-DEAS geometric model using the 3-D graphics hardware accelerator of the workstation. Therefore, CPU time for RCS prediction is spent only on the electromagnetic part of the computation, while the more time-consuming geometric model manipulations are left to the graphics hardware. This hybrid graphic-electromagnetic computing (GRECO) results in realtime RCS prediction for complex radar targets.
\end{abstract}

\section{INTRODUCTION}

$\mathrm{T}$ HE aim of this paper is focused on the prediction of monostatic radar cross section (RCS) of large and complex radar targets. The main objective of the algorithms presented here is obtaining real-time results for arbitrary and very general target shapes using a graphic workstation. Thus, the RCS prediction software can be easily integrated with the computer-aided design (CAD) package used to model the target, providing an efficient tool for interactive modeling, design, and analysis of aircraft with RCS specifications. In that context, what we understand by real time is fast enough to achieve interactive design of low-RCS shapes.

The main difficulty for RCS prediction of complex targets using high-frequency techniques is the computa-

Manuscript received January 28,1993 . This work was supported by the Spanish "Comisión Interministerial de Ciencia y Tecnología (CICYT) under Project ACCION ESPECIAL PRONTIC "Programa de investigación aplicada para el desarrollo y validación de métodos de cálculo numérico para la predicción y análisis de las características de los ecos radar (RCS) y su reducción," TIC 88-288E. Management: "Dirección General de Telecomunicaciones" (D.G. Tel). Coordination: C.A.S.A.

The authors are with the Antennas, Microwave and Radar Group, Department Teoria del Senyal i Communicacions, Universitat Politécnica de Catalunya, Apdo. 30002, 08080 Barcelona, Spain.

IEEE Log Number 9211257. tion of surface and line integrals over an arbitrary shape. This shape is defined by CAD geometric modeling software using either a facets and wedges approximation or a parametric surface approach. In both cases, the unit normal to the surface at each point of the geometric model must be obtained in order to perform the electromagnetic computations.

An additional problem is that, according to highfrequency theory, the currents are assumed to be zero over the surfaces or edges not illuminated by the incident wave-shadowed by other parts of the aircraft - so the surface or edge radiation integrals extend only over the region of the target illuminated by the incident field.

The classical high-frequency techniques for RCS prediction are based on a target model in terms of facets and wedges [2]-[4]. Using this approach, the identification of illuminated and shadowed regions on the geometrical model of the target is a difficult and very time consuming problem; therefore the classical high-frequency codes must dedicate considerable effort to the manipulation of the aircraft geometric model prior to the electromagnetic computations.

\section{Graphical Electromagnetic COMPUTING (GRECO)}

These difficulties are overcome by the new graphical processing technique: using a graphic workstation we can obtain a 3-D image of the target. If the viewpoint of the target is located at the position of the monostatic radar, then the picture on the workstation screen contains only the illuminated surfaces and edges: the shadowed ones are not visible from the observer viewpoint because they have been removed by the 3-D visualization hardware.

Furthermore, if the image is obtained using 3-D illumination and rendering, we can define the illumination source parameters in such a way that the red, green, and blue ( $R, G, B)$ components of the color of each pixel of the image are equal to the $\left(n_{x}, n_{y}, n_{z}\right)$ components of the unit normal to the surface at this point. The screen memory of the workstation has then six-dimensional information of each pixel: $x, y, z$ coordinates and the R, G, B color components, which are equal to $\left(n_{x}, n_{y}, n_{z}\right)$. Thus, the screen memory has available all the information needed in order to compute high-frequency approximations. This $x, y, z, n_{x}, n_{y}, n_{z}$ information is supplied in real time by the hardware graphics accelerator of the workstation provided that the target has been modeled with reasonable accuracy. In conclusion, the CPU run 
time involves only the electromagnetic part of the computation, leaving the geometric task to the graphics hardware.

As shown in Fig. 1, RCS is obtained by GRECO code in the following steps [6]-[11].

1) Geometric modeling of the aircraft with a CAD package.

2) Image of the target at the workstation screen obtained in real time by the graphics hardware accelerator.

3) $x, y, z, n_{x}, n_{y}, n_{z}$ coordinates of each point of the illuminated surface are obtained by graphical processing of the image.

4) The following high-frequency approximations are then computed:

- Reflection at perfectly conducting surfaces by PO approximation [1].

- Reflection at coated surfaces by PO and IBC approximations [1], [20], [21].

- Diffraction at edges by MEC and PTD [1] incremental length diffraction coefficients (ILDC) [22].

Steps 1) to 3) involve the geometric and graphical part of the computation and will be explained in detail in Section III, while step 4), the electromagnetic portion, will be treated more briefly in Section IV.

\section{GRAPHICAL Processing of AN IMAGE OF tHE TARGET}

\section{A. Target Geometric Modeling}

A computer-aided design (CAD) package for geometric modeling of solids [12] has been used for modeling target geometry. The aircraft is described either as a collection of facets and wedges or parametric surfaces. The last are defined using two-dimensional nonuniform rational $B$ splines (NURBS) [13], [14].

As stated before, classical RCS analysis packages usually describe the target in terms of facets and wedges [2]-[4]. However, parametric surfaces present the following advantages for both complex object modeling and RCS prediction and optimization.

- Complex objects require a very large number of facets, while only a few parametric surfaces. Thus, the parametric approach requires less information to define the model, which results in less mass storage memory and faster processing. Another important point is that the number of degrees of freedom for RCS optimization algorithms is also smaller with parametric surfaces.

- The faceting approach introduces artificial edges and vertices between facets, so that the surface presents a "faceted" appearance while the parametric surface is smooth and conforms precisely to the real one. For that reason, RCS computed from the faceted model contains the so-called facet noise, which is greatly

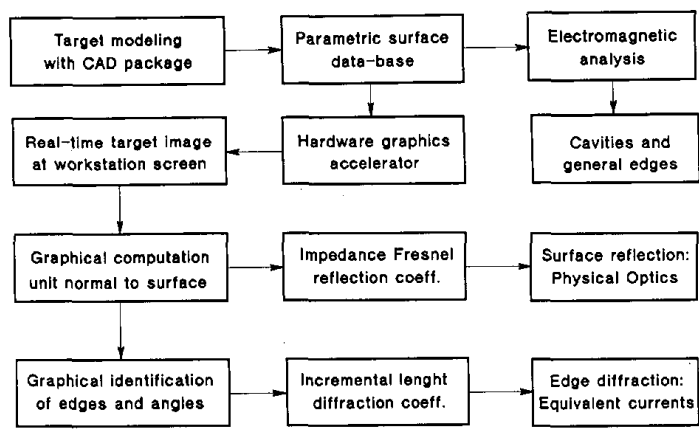

Fig. 1. Block diagram with the different steps for RCS prediction by the graphics electromagnetic computing (GRECO) code.

reduced, if not totally eliminated, when the parametric surface model is used instead.

\section{B. Real-Time Image of the Target}

Hardware graphics accelerators of high-performance workstations are able to render a 3-D visualization of a parametric surface model in real time. Shadowed parts of the scene are removed from the image by the graphics hardware, so that the picture at the workstation screen contains only the surfaces visible from the observer viewpoint.

As shown in Fig. 2, the input information for the graphics accelerator must be, at least [18]:

- Geometric model: NURB surface parameters.

- Rendering: Surface coefficients for specular and diffuse reflection, pattern of the reflected beam, etc.

- Illumination: Position, orientation, radiation pattern, and color of the light sources.

- Observer: 3-D viewpoint and direction of observation.

The six-dimensional output of the graphics accelerator for each pixel comprises the three $R, G$, and $B$ color components, the 2-D location on screen, and the distance to the observer. For example, in the old Hewlett-Packard Turbo SRX graphics accelerator used by GRECO, the coding of this information is: 2-D screen $1280 \times 1024$ pixels, distance to the observer is $16 \mathrm{~b}$ and $\mathrm{R}, \mathrm{G}, \mathrm{B}$ color components in $8 \mathrm{~b}$ each $\times 3=24 \mathrm{~b}$. However, much better resolution can be provided by the latest and more powerful graphics accelerators.

\section{Graphical Processing}

In this subsection we will use the following convention for the 3-D coordinate axis: $x, y$ are the 2-D coordinates of the workstation screen, respectively, in the horizontal and vertical directions, while $z$ is in the direction perpendicular to the screen. If the observer viewpoint is located on the monostatic radar position, the $z$ coordinate of each pixel is equal to the distance between the observer and each surface element.

From the electromagnetic point of view, this $z$ information is of substantial importance for adding coherently the high-frequency local contribution of each surface ele- 


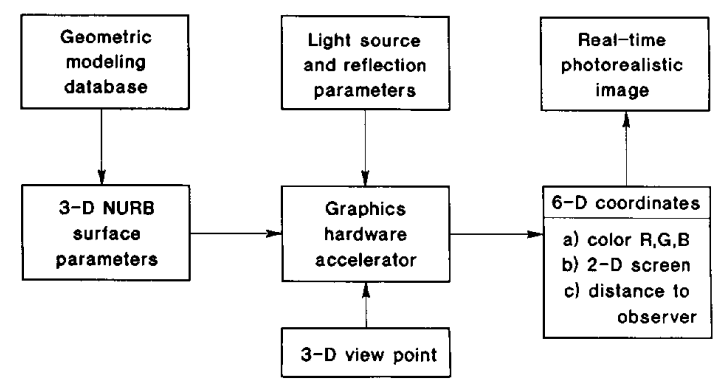

Fig. 2. Inputs and outputs of the hardware graphics accelerator.

ment. Using this convention, the six-dimensional output of the hardware graphics accelerator comprises the $x, y, z$, R, G, and B coordinates and color components for each pixel.

1) Shadowed and Eclipsed Surfaces Identification: As stated before, one of the main difficulties in computing the physical optics surface integral by classical techniques (faceting approach [2]-[4]) is the detection of shadowed regions. However, GRECO has no need to face this problem, because hidden surfaces of the image have been previously removed by the hardware graphics accelerator.

A very simple validation of the hidden surface elimination and the correctness of the $z$ coordinate information supplied by the graphics accelerator is the two-sphere system shown in Fig. 3. The RCS computed by GRECO has been normalized with respect to the RCS of only one sphere. It can be noticed that when one of the two spheres is shadowing the other one, aspect angle close to $90^{\circ}$, the RCS of the two-sphere system is equal to the RCS of only the visible one.

In Fig. 3, the lobe at $30^{\circ}$ does not equal the RCS of the two spheres. The reason is that numerical computation of PO surface integral for a general curved surface always introduces some error, because the contribution from the region of fast phase variation, that should cancel out according to the stationary phase principle, is very sensible to the surface discretization and in fact does not cancel out. This error is smaller in GRECO code than in the facets and wedges approach due to the better discretization of the parametric surfaces, and can be further improved by using a $\cos ^{n} \theta$ taper function (see (7) in Section IV-A).

2) Computation of Unit Normal to Surface: If the scene is rendered using the Phong local illumination model [19], the color of each pixel depends only on the normal to the surface element associated to this pixel and on the location of the observer and light sources. As the position of both the observer and light sources is known, for each pixel of the image it is possible to obtain the normal to the surface from the color information.

According to the Phong illumination model, when the surface reflection is diffuse, the brightness of a pixel is computed separately for each $R, G, B$ color using the equation

$$
L_{d}=L_{s} \alpha_{L} \rho_{d} \hat{n} \cdot \hat{r}_{i}
$$

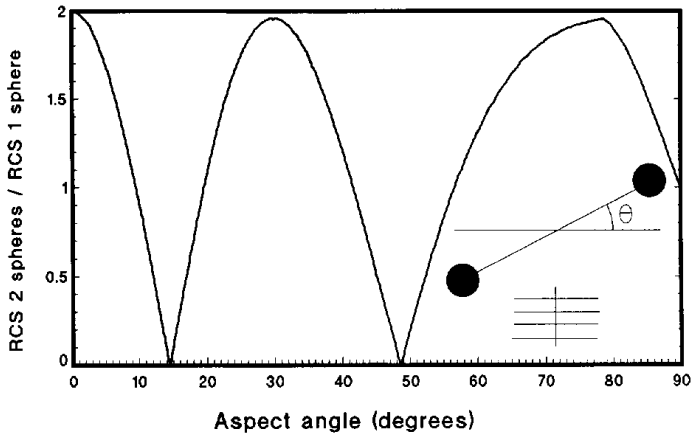

Fig. 3. Two-sphere system for visualizing the shadowing effects in GRECO, using the hidden surface removal capabilities of the graphics accelerator. The distance between the spheres is $\lambda$ and the radius $0.1 \lambda$. The RCS of the two-sphere system is normalized with respect to the RCS of only one sphere. The shadowing effect is important when the aspect angle is close to $90^{\circ}$ : the normalized RCS of the system approaches to one as the first sphere eclipses the second one.

where $L_{d}$ is the diffuse brightness for each color, $L_{s}$ the intensity of the light source, $\alpha_{L}$ the attenuation of light propagation in the medium, $\rho_{d}$ is the diffuse reflection coefficient, $\hat{n}$ is the unit normal to surface at this pixel, and $\hat{r}_{i}$ is the unit vector along the direction of incidence (see Fig. 4).

If in (1) coefficients $L_{s}, \alpha_{L}, \rho_{d}$ are equal to unity, we can compute the brightness of the pixel as the projection $\hat{n} \cdot \hat{r}_{i}$ of the unit normal to the surface on the direction of illumination (see Fig. 4). Therefore, for three light sources of purely green, red, and blue colors, respectively, located over each one of the three coordinate axis, the three color components for this pixel are equal to the $\left(n_{x}, n_{y}, n_{z}\right)$ components of the unit normal to surface

$$
\begin{array}{r}
\text { RED: } \hat{r}_{i}=\hat{x} \Rightarrow R=\hat{n} \cdot \hat{x}=n_{x} \\
\text { BLUE: } \hat{r}_{i}=\hat{y} \Rightarrow B=\hat{n} \cdot \hat{y}=n_{y} \\
\text { GREEN: } \hat{r}_{i}=\hat{z} \Rightarrow G=\hat{n} \cdot \hat{z}=n_{z} \\
\hat{n}=\left(n_{x}, n_{y}, n_{z}\right)
\end{array}
$$

Fig. 5 shows an image of F-117 stealth aircraft illuminated according to (2). Thus, purely red color means that the unit normal to surface is horizontal $(x)$, blue color vertical $(y)$, and green color perpendicular to the screen $(z)$. When the unit normal is not parallel to any of the three coordinate axis, the blending of the three color components (Red, Blue, Green) is equal to the $\left(n_{x}, n_{y}, n_{z}\right)$ components of the unit normal.

As the color components are always positive quantities, there is an ambiguity in the sign of the $\left(n_{x}, n_{y}, n_{z}\right)$ components of the unit normal. To obtain only the positive values, the graphics accelerator must display only the illuminated surfaces, with $\cos \theta_{i}>0$, and remove the backfacing ones, with $\cos \theta_{i}<0$. In order to obtain positive and negative values for $\left(n_{x}, n_{y}, n_{z}\right)$, it is necessary to illuminate the target from both the positive and negative axis directions, using different light sources. As we need a 


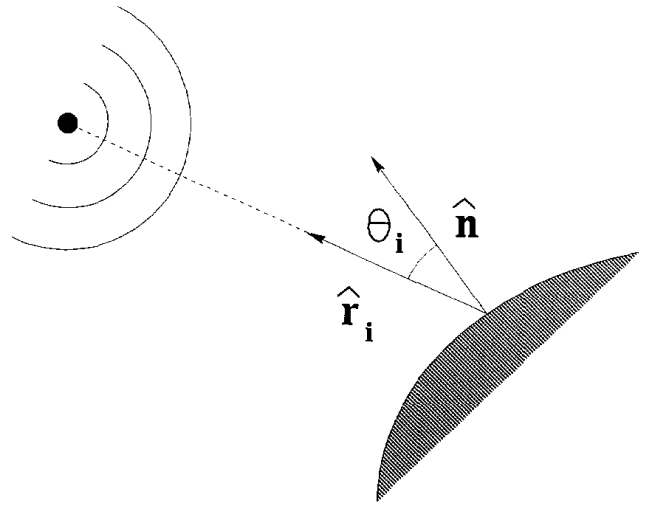

Fig. 4. Geometry for diffuse reflection according to Phong illumination model.

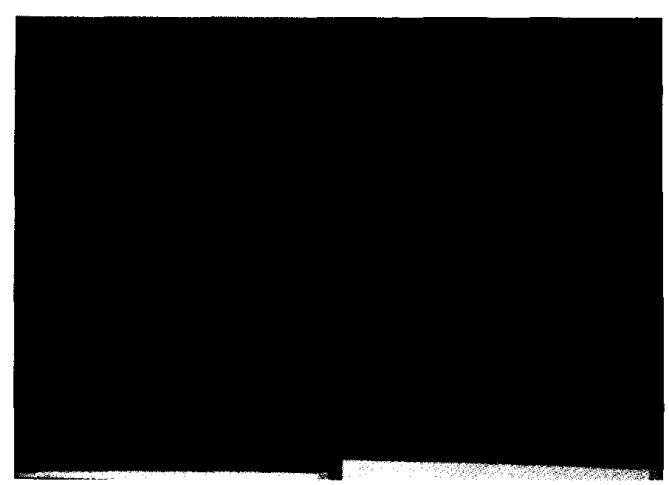

Fig. 5. Rendered image of aircraft F-117 at the workstation screen. Three red, blue, and green light sources are located on $x, y, z$ axis, respectively. The blending of the three colors at each pixel is equal to the $\left(n_{x}, n_{y}, n_{z}\right)$ components of the unit normal.

total of six light sources in order to obtain the six positive and negative values of $\left(n_{x}, n_{y}, n_{z}\right)$, and there are only three independent color components (R, G, B) two different three-color images must be displayed separately. Fig. 6 shows the two images of the generic missile defined by $\mathrm{N}$. Youssef in [2].

\section{ELECTROMAGNETIC COMPUTING}

Electromagnetic computing, the second main step of GRECO, uses as input information the $x, y, z$ coordinates and the $n_{x}, n_{y}, n_{z}$ unit normal of each illuminated pixel of the target. From the knowledge of this input information, a number of different high-frequency approximations can be implemented in a way completely independent of the target geometry. As high-frequency theory for RCS prediction is well known [1], [20], we will concentrate on the efficient implementation of high-frequency techniques in GRECO code.

\section{A. Physical Optics}

According to the physical optics (PO) technique, monostatic RCS of a perfectly conducting surface can be ap-

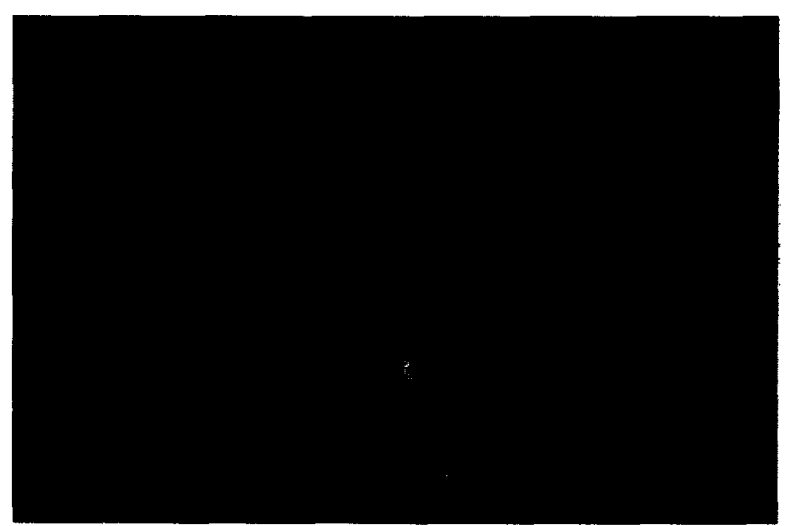

(a)

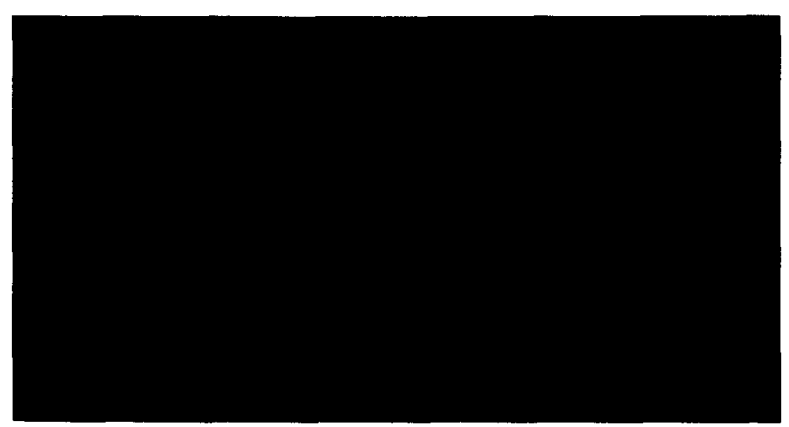

(b)

Fig. 6. Rendered images of generic missile model defined in [2]. Six red, blue, and green light sources are located on positive and negative $x, z$ axis (first image) and $y, z$ axis (second image). The blending of the three colors at each pixel of the two images is equal to the positive and negative $\left(n_{x}, n_{y}, n_{z}\right)$ components of the unit normal.

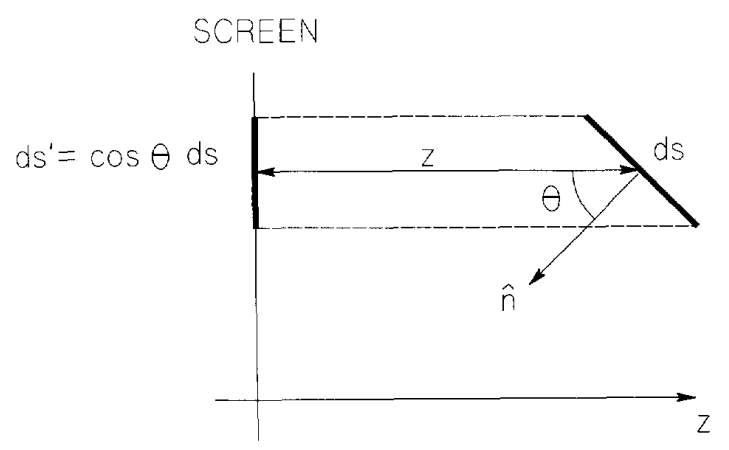

Fig. 7. Surface element and its projection on the workstation screen.

proximated in high frequency by the expression [20]

$$
\sigma=\frac{4 \pi}{\lambda^{2}}\left|\int_{s} \cos \theta e^{2 j K z} d s\right|^{2}
$$

where $\theta$ is the angle between the normal to the surface and the direction of incidence, and $z$ is the distance from the differential of surface $d s$ to the observer projected on the incidence direction. The surface integral extends only over the region illuminated by the incident wave. 
However, the image of the target processed by GRECO is actually a projection on the workstations screen of the real 3-D surface (see Fig. 7), so that the differential of surface on the screen $d s^{\prime}$-equivalent to one pixel-is equal to $d s^{\prime}=\cos \theta d s$ and the PO surface integral (3) can be written as

$$
\sigma=\frac{4 \pi}{\lambda^{2}}\left|\int_{s} \cos \theta e^{2 j K z} d s\right|^{2}=\frac{4 \pi}{\lambda^{2}}\left|\int_{\mathrm{SCREEN}} e^{2 j K z} d s^{\prime}\right|^{2}
$$

Discrete computation of surface integral (4) lead to

$$
\sigma=\frac{4 \pi}{\lambda^{2}}\left|\sum_{\text {PIXELS }} e^{2 j K z}\right|^{2}
$$

which is the equal to the coherent addition of the phase contribution from all the pixels in the target image. This phase contribution is due to the distance $z$ from each pixel to the observer.

It must be noted that (5) is correct only if a pixel radiates as an infinitesimal aperture, i.e., it is equivalent to the projection on the screen of a differential of surface $d s$ much smaller than a wavelength. If the number of pixels in the screen is large enough, this condition is usually accomplished. However, when the incidence is grazing over the surface, $\theta \rightarrow 90^{\circ}$, the projection $d s^{\prime}$ on the screen-one pixel is very small, but the surface $d s$ may be very large.

Accordingly, in general we cannot assume that each pixel radiates as an infinitesimal aperture, but as an electrically large one. Assuming that one pixel is equivalent to a rectangular aperture with uniform illumination, its contribution to the far fields can be approximated by a sinc function of the angle $\theta$, and the PO surface integral becomes in the discrete domain:

$$
\sigma=\frac{4 \pi}{\lambda^{2}}\left|\sum_{\text {PIXELS }} \operatorname{sinc}\left(K \frac{l}{\cos \theta} \operatorname{sinc} \theta\right) e^{2 j K z}\right|^{2}
$$

where $l$ is the size of a square pixel $d s^{\prime}$ in the screen and $l / \cos \theta$ is the size of the $d s$ which has been projected on this pixel.

A problem frequently arising when computing the RCS of curves surfaces using the physical optics approximation is the presence of spurious oscillations in an RCS versus frequency plot. These oscillations are due to the contribution of the abrupt transition in the equivalent currents at the boundary between the illuminated and the shadowed regions [1].

According to high-frequency theory, the contribution to the monostatic RCS from a curved surface comes from the specular reflection point, where the normal to the surface is closed to the incidence direction, $\theta \rightarrow 0^{\circ}$ in Fig. 4. As mentioned in the comments to Fig. 3 (Section III.C.1) the contribution from the region of fast phase variation, close to the boundary between illuminated and shadowed regions, should cancel out, but it does not, due to the error in the surface discretization. As in the shadow boundary we have $\theta \rightarrow 90^{\circ}$, this spurious contribution can

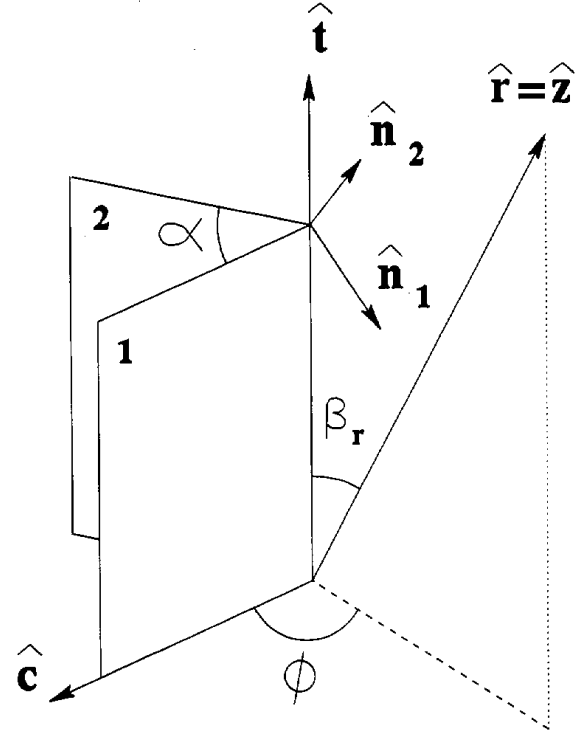

Fig. 8. Wedge geometry. The direction of incidence and observation is along $z$-axis.

be removed without modifying the specular contribution if the contribution from each pixel is multiplied by a $\cos ^{n} \theta$ taper function:

$$
\sigma=\frac{4 \pi}{\lambda^{2}}\left|\sum_{\mathrm{PIXELS}} \cos ^{n} \theta \operatorname{sinc}(K l \tan \theta) e^{2 j K z}\right|^{2}
$$

where $n$ is a parameter that controls the effect of this stationary phase approximation. When $n=0$, the PO surface integral is computed rigorously over the discretized surface and, on the other hand, when $n>1$ significant errors in the sidelobes level of flat plates may be introduced.

Equations (6) or (7) can be implemented very efficiently in GRECO, because the $\cos \theta$ and sinc functions depend only on the angle $\theta$. Using the illumination sources described in section III.C.2-see F-117 in Fig. 5-, the Green color component of each pixel is equal to

$$
\operatorname{GREEN}=n_{z}=\cos \theta
$$

As the green color is codified in $8 \mathrm{~b}$, the $\cos ^{n} \theta$ $\operatorname{sinc}(K l \tan \theta)$ function can be tabulated in a 256 entry table indexed by the Green color component of each pixel. If the $e^{-2 j K z}$ phase exponential is also tabulated in a $2^{16}$ entry table indexed by coordinate $z$, we can add the contribution from each pixel to the PO integral with only two floating-point real-number multiplications and additions.

\section{B. Impedance Boundary Condition}

Radar-absorbent coatings are considered through impedance boundary condition (IBC) and physical optics approximations, which lead to a very simple formulation of PO surface integral. It must be noticed that the PO + 
IBC approach is valid only for surface reflection, when a specular reflection point exists. Impedance wedges should be treated by a higher order approximate boundary condition.

According to IBC, the contribution from each pixel in (7) must be multiplied by Fresnel reflection coefficients for polarizations parallel $\Gamma_{\|}$and perpendicular $\Gamma_{\mid}$to the plane of incidence:

$$
\begin{aligned}
\sigma=\frac{4 \pi}{\lambda^{2}} \mid \sum_{\mathrm{PIXELS}}\left[\Gamma_{\mid} E_{\|}^{i} \hat{e}_{\|}^{i}\right. & \left.+\Gamma_{\mid} E_{\mid}^{i} \hat{e}_{\mid}^{i}\right] \\
& \left.\cos \theta \operatorname{sinc}(K l \tan \theta) e^{2 j K z}\right|^{2}
\end{aligned}
$$

where $E_{\|}^{i}$ and $E_{\mid}^{i}$ are, respectively, the components of the incident field in the directions parallel and perpendicular to the plane of incidence, $e_{\|}^{i}$ and $e^{i}$. Separation of the incident field into parallel and perpendicular components lead to the following expressions for the copolar and crosspolar polarizations [11]:

Vertical copolar polarization:

$\sigma_{y y}=\frac{4 \pi}{\lambda^{2}} \mid \int_{s} \cos \theta \operatorname{sinc}(K l \tan \theta) \cdot\left[\frac{\Gamma_{\|} n_{y}^{2}+\Gamma_{\mid} n_{x}^{2}}{n_{x}^{2}+n_{y}^{2}}\right] e^{2 j K z} d s^{\prime}$

Horizontal copolar polarization:

$$
\begin{aligned}
\sigma_{x x}=\frac{4 \pi}{\lambda^{2}} \mid \int_{s} \cos \theta \operatorname{sinc}( & K l \tan \theta) \\
\cdot & \left.\cdot\left[\frac{\Gamma_{\|} n_{x}^{2}+\Gamma_{\mid} n_{y}^{2}}{n_{x}^{2}+n_{y}^{2}}\right] e^{2 j K z} d s^{\prime}\right|^{2}
\end{aligned}
$$

Crosspolar polarization:

$$
\begin{aligned}
& \sigma_{x y}=\frac{4 \pi}{\lambda^{2}} \mid \int_{s} \cos \theta \operatorname{sinc}(K l \tan \theta) \\
&\left.\cdot\left[\frac{\left(\Gamma_{\|}-\Gamma_{1}\right) n_{y} n_{x}}{n_{x}^{2}+n_{y}^{2}}\right] e^{2 j K z} d s^{\prime}\right|^{2}
\end{aligned}
$$

where $n_{x}, n_{y}, n_{z}$ are, respectively, the components of the unit normal to the surface along the horizontal, vertical, and incidence directions. Using the illumination scheme presented in Section III.C. $2, n_{x}, n_{y}, n_{z}$ at each $d s^{\prime}$ are equal to the $R, G, B$ color components of the pixel corresponding to this $d s^{\prime}$ (see Figs. 5 and 6).

Formulation of Fresnel reflection coefficients as a function of equivalent surface impedance is well known and can be found in [1], [20], [21], etc. Surface impedance of a dielectric coating over a perfect conducting surface is obtained in the usual way through a transmission line equivalent circuit.

As reflection coefficients ae dependent only on $\theta$ angle, which according to (8) is related to the green color component of each pixel in the image-see Fig. 5-the paral- lel and perpendicular reflection coefficients can be tabulated in a 256 entry table indexed by the green color component, thus avoiding the reflection coefficients for each pixel.

\section{Method of Equivalent Currents (MEC)}

According to high-frequency theory, the far-field scattered from a wedge can be assumed as radiated by an equivalent line current located on the edge [1], [20]. This equivalent current depends on both the directions of incidence and observation relative to orientation of the edge, so that its value is not constant along the edge. Equivalent currents are usually expressed as a function of some incremental length diffraction coefficients (ILDC) [22]. The monostatic far-field scattering from the wedge, resulting from the radiation of equivalent currents, is [1]

$$
\begin{array}{r}
E^{r}=E_{0} \frac{e^{-j K r}}{2 \pi r} \int_{\mathrm{EDGE}}\left[-D_{\|} \sin \gamma \hat{e}_{\|}^{i}-D_{x} \cos \gamma \hat{e}_{\|}^{i}\right. \\
\left.-D_{\mid} \cos \gamma \hat{e}_{\mid}^{i}\right] e^{2 j K z} d l^{\prime}
\end{array}
$$

where the line integral extends along the edges illumi$\left.\right|^{2}$ nated by the incident wave, $e_{\|}^{i}$ and $e_{\mid}^{i}$ are, respectively, the unit vectors parallel and perpendicular to the plane of incidence-which is defined by the incidence $\hat{z}$ and edge $t$ directions - and $\gamma$ is the angle between the incident electric field and $e^{i}$.

In (13), $D_{\|}, D_{\mid}, D_{x}$ stand for the monostatic ILDC's, which express the dependence of the scattered field on the angles $\alpha, \phi, \beta_{r}$ defined in Fig. 8. The formulation of the ILDC and references to the original papers of Keller (GTD), Ufimtsev (PTD), Michaeli (MEC), and Mitzner (ILDC) can be found in [1], [11], and [20].

Separation of the incident field of (13) into parallel and perpendicular components leads to the following expressions for the copolar and crosspolar polarizations [11]:

Copolar horizontal:

$$
\begin{aligned}
E_{x x}^{s}=E_{0} \frac{e^{-j K r}}{2 \pi r} \int_{\text {EDGE }} & {\left[-D_{\|} \frac{t_{x}^{2}}{t_{x}^{2}+t_{y}^{2}}\right.} \\
& \left.+D_{x} \frac{t_{x} t_{y}}{t_{x}^{2}+t_{y}^{2}}-D_{\mid} \frac{t_{y}^{2}}{t_{x}^{2}+t_{y}^{2}}\right] e^{2 j K z} d l^{\prime}
\end{aligned}
$$

Copolar vertical:

$$
\begin{aligned}
E_{y y}^{s}=E_{0} \frac{e^{-j K r}}{2 \pi r} \int_{\mathrm{EDGE}} & {\left[-D_{\|} \frac{t_{y}^{2}}{t_{x}^{2}+t_{y}^{2}}\right.} \\
& \left.-D_{x} \frac{t_{x} t_{y}}{t_{x}^{2}+t_{y}^{2}}-D_{\mid} \frac{t_{x}^{2}}{t_{x}^{2}+t_{y}^{2}}\right] e^{2 j K z} d l^{\prime}
\end{aligned}
$$

Crosspolar horizontal transmission-vertical reception:

$$
\begin{aligned}
E_{x y}^{s}= & E_{0} \frac{e^{-j K r}}{2 \pi r} \int_{\text {EDGE }}\left[+D_{\|} \frac{t_{x} t_{y}}{t_{x}^{2}+t_{y}^{2}}\right. \\
& \left.-D_{x} \frac{t_{y}^{2}}{t_{x}^{2}+t_{y}^{2}}-D \frac{t_{x} t_{y}}{t_{x}^{2}+t_{y}^{2}}\right] e^{2 j K z} d l^{\prime}
\end{aligned}
$$


Crosspolar vertical transmission-horizontal reception:

$$
\begin{aligned}
E_{y x}^{s}= & E_{0} \frac{e^{-j K r}}{2 \pi r} \int_{\mathrm{EDGE}}\left[-D_{\|} \frac{t_{x} t_{y}}{t_{x}^{2}+t_{y}^{2}}\right. \\
& \left.-D_{x} \frac{t_{x}^{2}}{t_{x}^{2}+t_{y}^{2}}+D_{\mid} \frac{t_{x} t_{y}}{t_{x}^{2}+t_{y}^{2}}\right] e^{2 j K z} d l^{\prime}
\end{aligned}
$$

where $t=\left(t_{x}, t_{y}, t_{z}\right)$ is the unit vector parallel to $d \overrightarrow{l^{\prime}}$, and the $x, y, z$ axis have been defined as, respectively, the horizontal, vertical, and incidence directions.

In order to compute MEC line integrals (14)-(17), GRECO code must obtain the ILDC for each pixel of the image laying along an edge, and add coherently the contributions from all the pixels. As the ILDC depend on the angles $\alpha, \phi, \beta_{r}$, defined in Fig. 8, GRECO must first compute these angles from the knowledge of the unit normal to both faces of the edge [11]

$$
\begin{gathered}
\alpha=\cos ^{-1}\left(-\hat{n}_{1} \cdot \hat{n}_{2}\right) \\
\sin \beta_{r}=|\hat{r} \times \hat{t}|=\sqrt{t_{x}^{2}+t_{y}^{2}} \\
\cos \phi=\frac{n_{1 x} t_{y}-n_{1 y} t_{x}}{\sqrt{t_{x}^{2}+t_{y}^{2}}}
\end{gathered}
$$

where $t$ is the unit vector along the edge direction

$$
t=\frac{\hat{n}_{1} \times \hat{n}_{2}}{\left|\hat{n}_{1} \times \hat{n}_{2}\right|} .
$$

As the reflection at the (curved or flat) faces of the wedge has been already obtained in Section IV.A using Physical Optics, now we must compute only the contribution to far-field scattering from the edge alone. This can be achieved using Ufimtsev PTD or Mitzner ILDC coefficients [1], [11], [20]. The former are valid only when the observation direction lays on the Keller cone [1], [11], [20], while the later are valid for an arbitrary bistatic observation.

However, for backscattering monostatic RCS problems, it can be easily shown [11] that the Mitzner and PTD coefficients for polarization parallel or perpendicular to the plane of incidence, $D_{\|}$and $D_{1}$, respectively, are equal; while the $D_{x}$ ILDC is assumed to be zero in Ufimtsev PTD. This last assumption implies that there are no cross-polarization effects when the incident field is perpendicular to the plane of incidence.

Although the exact ILDC's for scattering from edges without surface reflection at faces are Mitzner ILDC's, in GRECO code we have implemented physical theory of diffraction (PTD) coefficients. This is actually a very good approximation when the incidence direction is near the plane perpendicular to the edge [11], and it is well known that the RCS flashes from the edge scattering are produced by normal incidence, while the overall contribution from oblique incidence is usually negligible.

Figs. 9 and 10 show, respectively, the monostatic PTD coefficients [11] for polarization parallel $\left(f=D_{\|}\right)$and

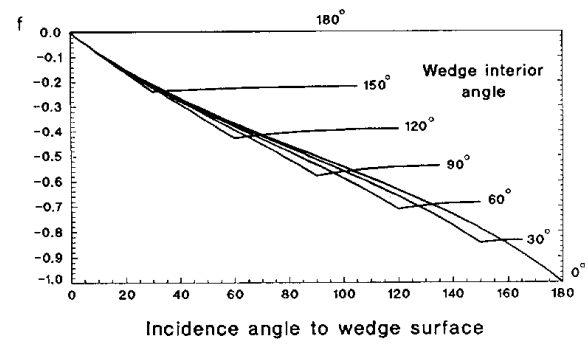

Fig. 9. Monostatic PTD coefficient $f=D_{\text {f }}$ for polarization parallel to the plane of incidence, versus the angle $\phi$ between incidence direction and wedge surface, as defined in Fig. 9.

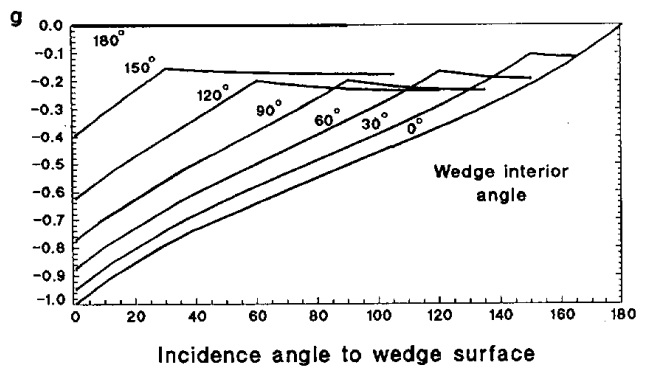

Fig. 10. Monostatic PTD coefficient $g=D_{1}$ for polarization perpendicular to the plane of incidence, versus the angle $\phi$ between incidence direction and wedge surface, as defined in Fig. 9.

perpendicular $(g=D)$ to the plane of incidence, versus the angle $\phi$ between incidence direction and wedge surface, as defined in Fig. 8. The expressions for PTD coefficients can be found in [1], [11], [20], etc. These equations involve several trigonometric functions, so that the exact computation of PTD coefficients for each pixel of the target laying on an edge must be avoided. For that reason, we have developed the following linear approximation to monostatic PTD coefficients, as plotted in Figs. 9 and 10:

$$
\begin{array}{cc}
1 \text { face visible } & 2 \text { faces visible } \\
0 \leq \phi \leq \pi-\alpha & \pi-\alpha \leq \phi \leq \pi-\alpha / 2 \\
f \approx-\frac{\phi}{\pi} & f \approx-(n-1) \\
g \approx-\left(\frac{n}{2}-\frac{\phi}{\pi}\right) & g \approx \tan \frac{\alpha}{2}-\frac{2}{n} \cot \frac{\pi}{n}
\end{array}
$$

where $n=(2 \pi-\alpha) / \pi$ is the exterior wedge angle normalized by $\pi$.

In summary, GRECO code computes high-frequency scattering from edges by the method of equivalent currents (MEC) in the following steps:

1) An image of the target is made at the workstation screen. Hidden edges are removed by the graphics accelerator hardware, so that only visible ones are displayed.

2) The surface unit normal at each pixel of the image is computed by graphical processing of the image (see Section III.C.2). 
3) Edges are detected on the target image as discontinuities of the unit normal to the surface when the $z$ coordinate remains continuous. It must be noted that eclipsed surfaces may also produce discontinuities in the normal to the surface (see Fig. 3) but in that case the $z$ coordinate would be discontinuous.

4) For each pixel along the detected edges, $\alpha, \phi, \beta_{r}$ are computed from the unit normal to each face of the wedge using (18)-(21). Monostatic PTD diffraction coefficients are then obtained using a very simple linear approximation (22), (23), which saves run-time avoiding the computation of trigonometrical functions.

5) Line integrals (14)-(17) of MEC are evaluated adding coherently the PTD coefficients for each pixel.

The phase center of the ILDC edge is assumed to be the same that the phase center of one of the adjacent pixels, which is not on the edge. The error is usually very small because the $z$ coordinate (distance to the observer) is discretized in at least $2^{16}$ samples. However, for grazing incidence this approximation is improved by computing the $z$ coordinate of the edge from the $z$ and $\hat{n}$ information at the adjacent pixels.

\section{Results for Complex OBJects}

The graphical electromagnetic computing technique presented in this paper has been validated comparing the results for simple and canonical objects with analytical solutions [11]. Results for complex radar targets can be found in [6]-[11], and show good agreement with both measurements and faceting approach codes.

In this section, we will only present the results obtained by GRECO for the generic missile and NACA airfoil defined by N. Youssef in his classical paper [2], together with the result for the airfoil section defined in JINA'90 workshop [23].

Using an old Hewlett-Packard 380 workstation-Motorola $68040 \mathrm{CPU}$ with speed of only 2.5 Mflops-and a Turbo SRX graphics accelerator, we can compute the RCS of a complex aircraft in $0.2 \mathrm{~s} /$ angle (PO) or about 5-10 s/angle (MEC). The speed should be improved by a factor of 5 or 10 using the new and faster HP-700 RISC workstations with CRX 48Z graphics accelerator.

\section{A. Generic Missile}

Fig. 11 shows the geometry of the generic missile defined in [2] and presents the results of GRECO (only Physical Optics) compared with the prediction of TOTAL code [3] [4], developed at the University of Cantabria, Spain. TOTAL code is based on a facets and NURBS model, and takes into account surface reflection, edge diffraction, surface-surface, and surface-edge interactions. The results of RECOTA code and the measurements performed by Boeing Aerospace can be found in [2].

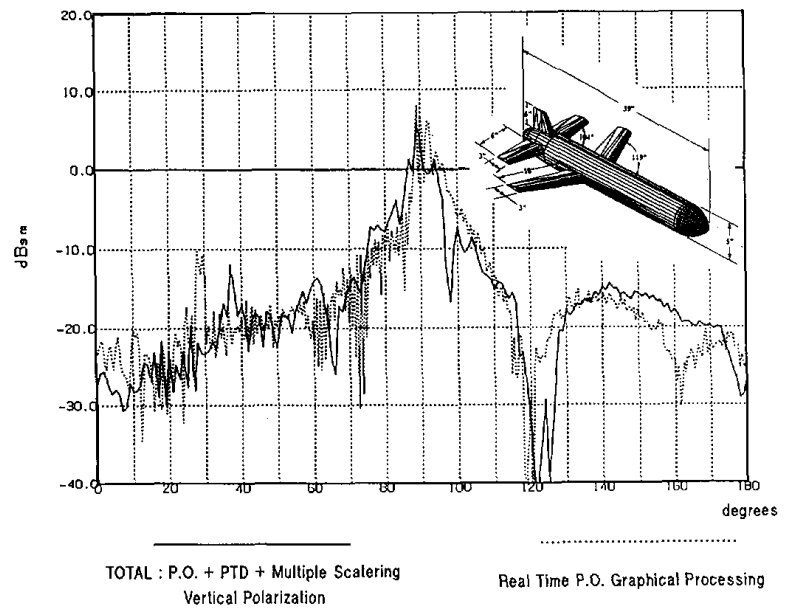

Fig. 11. Generic missile model defined in [2]. The fuselage length is about $40 \lambda$ at $12 \mathrm{GHz}$. Results of GRECO (only Physical Optics) compared with predictions of TOTAL [3] [4], at $12 \mathrm{GHz}$.

Table I compares the monostatic RCS results of GRECO, TOTAL, and RECOTA with measurements performed by Boeing Aerospace [2] at a frequency of 12 $\mathrm{GHz}$ and with vertical polarization. The agreement between the three predictions and the measurements is good, except for the flash produced by the leading edge of wing in TOTAL prediction. The reason for this discrepancy is an error in the missile geometric model used by TOTAL code.

Physical optics results of GRECO agree well with measurements because the vertical polarization diffraction at the trailing edges is negligible. In general, we have noticed that first-order PO approximation usually predicts with reasonable accuracy RCS of nonstealth radar targets -for example Boeing 727 [6]-[11] — so that the computation of edge diffraction and multiple interactions is not always necessary.

\section{B. Airfoil Sections}

As mentioned in [2], scattering form airfoils presents an interesting problem from the RCS analyst's point of view, because the validity of $\mathrm{PO}$ is tested at the leading edge (surface with small radius of curvature) and PTD at the trailing edge (sharp wedge). Although the validity of these approximations has been confirmed in [2], the graphical processing implementation must also be tested. For that reason, the results of GRECO code have been compared with those from RECOTA for the NACA 3317 airfoil [24] presented in [2].

Fig. 12 shows the results of GRECO code, while the results of RECOTA and the measurements performed by Boeing Aerospace can be found in [2]. The Table II compares the results of GRECO and RECOTA with the measurements:

It can be observed that GRECO results agree well with both measurements and the more rigorous PTD implementation of RECOTA [2]. This result validates the linear 
TABLE I

\begin{tabular}{|c|c|c|c|c|}
\hline & Measurement & RECOTA & TOTAL & GRECO \\
\hline $\begin{array}{c}\text { Leading edge } \\
\text { horizontal stabilizer }\end{array}$ & $\begin{array}{c}16^{\circ} \\
-19 \mathrm{dBsm}\end{array}$ & $\begin{array}{c}13^{\circ} \\
-17 \mathrm{dBsm}\end{array}$ & & $\begin{array}{c}11^{\circ} \\
-21 \mathrm{dBsm}\end{array}$ \\
\hline $\begin{array}{l}\text { Leading edge } \\
\text { of wing }\end{array}$ & $\begin{array}{c}29^{\circ} \\
-13 \mathrm{dBsm}\end{array}$ & $\begin{array}{c}29^{\circ} \\
-12 \mathrm{dBsm}\end{array}$ & $\begin{array}{c}37^{\circ} \\
-12 \mathrm{dBsm}\end{array}$ & $\begin{array}{c}29^{\circ} \\
-11 \mathrm{dBsm}\end{array}$ \\
\hline $\begin{array}{l}\text { Fuselage and } \\
\text { vertical stabilizer }\end{array}$ & $\begin{array}{c}89^{\circ} \\
8 \mathrm{dBsm}\end{array}$ & $\begin{array}{c}90^{\circ} \\
9 \mathrm{dBsm}\end{array}$ & $\begin{array}{c}89^{\circ} \\
8 \mathrm{dBsm}\end{array}$ & $\begin{array}{c}91^{\circ} \\
8 \mathrm{dBsm}\end{array}$ \\
\hline RCS null & $124^{\circ}$ & $131^{\circ}$ & $122^{\circ}-125^{\circ}$ & $119^{\circ}$ \\
\hline Angles $140^{\circ}-150^{\circ}$ & $-18 \mathrm{dBsm}$ & $-20 \mathrm{dBsm}$ & $-15 \mathrm{dBsm}$ & $-17 \mathrm{dBsm}$ \\
\hline
\end{tabular}
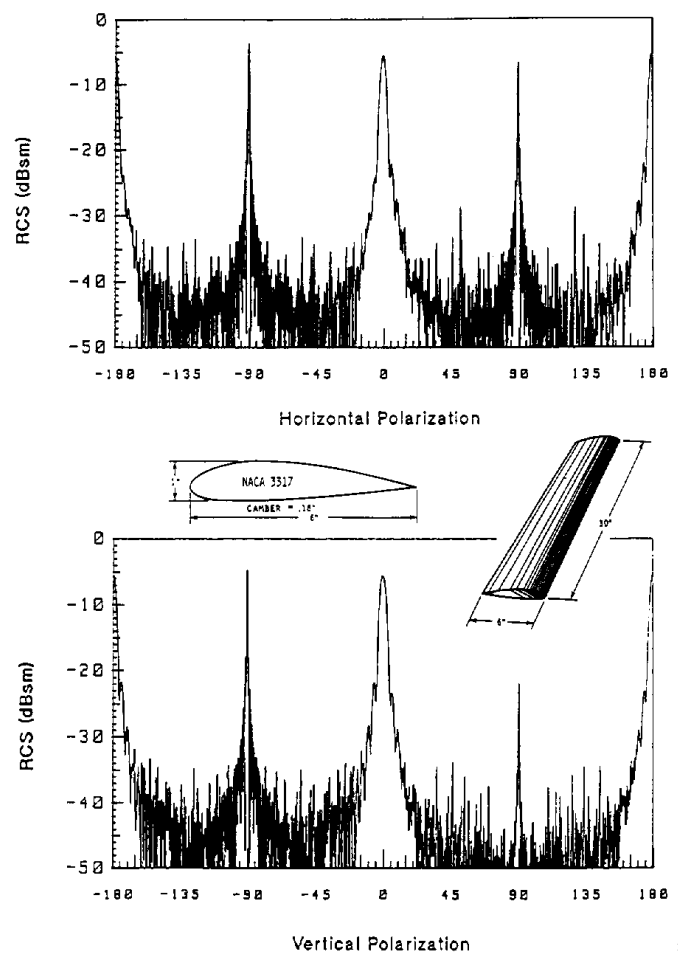

Fig. 12. Naca 3317 airfoil geometry as presented in [2]. GRECO monostatic RCS prediction for NACA 3317 airfoil at $16 \mathrm{GHz}$. The RCS peaks at $0^{\circ}$ and $180^{\circ}$ are produced by the flat tip of the airfoil, while the $90^{\circ}$ and $-90^{\circ}$ peaks are produced, respectively, by the leading and trailing edges.

TABLE II

\begin{tabular}{ccccc}
\hline & Polarization & Measurement & RECOTA & GRECO \\
\hline Leading edge & Horizontal & $-2 \mathrm{dBsm}$ & $-1 \mathrm{dBsm}$ & $-3 \mathrm{dBsm}$ \\
$\theta=90^{\circ}$ & Vertical & $-3 \mathrm{dBsm}$ & $-1 \mathrm{dBsm}$ & $-4 \mathrm{dBsm}$ \\
Trailing edge & Horizontal & $-8 \mathrm{dBsm}$ & $-6 \mathrm{dBsm}$ & $-7 \mathrm{dBsm}$ \\
$\theta=-90^{\circ}$ & Vertical & $-17 \mathrm{dBsm}$ & $-17 \mathrm{dBsm}$ & $-22 \mathrm{dBsm}$ \\
\hline
\end{tabular}

approximation of PTD coefficients (22), (23) and the graphical processing implementation of GRECO. The only significant differences are present at the trailing edge response for polarization perpendicular to the edge.

Some results of GRECO code were presented at the workshop "RCS of perfectly conducting or coated bodies" [23] held at Nice in November 1990. In this paper we will only refer to the two-dimensional generic airfoil section defined in problem no. 3 of the workshop [Fig. 13(a)].

Fig. 13(b) and (c) shows radar cross-width (RCW) predictions for a perfectly conducting airfoil surface, while in Fig. 13(d) and (e) a lossy dielectric layer has been considered. The coating has a relative dielectric permittivity $\epsilon_{r}=7.4-j 1.11$, relative magnetic permeability $\mu_{r}=1.4$ $-j 0.672$ and thickness $d=0.06 \lambda_{0}$, being $\lambda_{0}$ the wavelength in the vacuum.

GRECO high-frequency prediction is compared with a numerical method solution presented at the workshop by the Centre Commun de Tech. Louis-Bleriot of AEROSPATIALE.

It can be noticed in Fig. 13(b) and (c) that PO results predict correctly surface reflection, even for the leading edge at $0^{\circ}$, which has a small radius of curvature, but cannot predict edge diffraction when incident polarization is parallel to the trailing edge (TM) at $180^{\circ}$. However, if the method of equivalent currents with PTD coefficients is added to PO, the high-frequency result of GRECO agrees very well with the numerical solution of AEROSPATIALE.

It is important to notice that in Fig. 13(d) and (e) the PO + IBC high-frequency solution is correct only for aspect angles between $-120^{\circ}$ and $110^{\circ}$, which correspond to the specular reflection at the airfoil surface. Diffraction at the trailing edge should be analyzed by an impedance wedge solution in order to improve high-frequency results.

\section{CONCLUSIONS}

In this paper we have presented a new implementation of the well-known high-frequency techniques. Using a 3-D workstation with a graphics hardware accelerator, monostatic RCS prediction can be obtained potentially in real time for most reasonable large and complex radar targets.

Graphical electromagnetic computing (GRECO) has the following advantages over classical techniques [2]-[4]:

- Target can be modeled by parametric NURB surfaces, requiring less mass storage memory than the faceting approach, and adjusting more accurately to the real target surface, thus avoiding the "facet noise" usually present in classical facet modeling codes.

- Hardware graphics accelerator removes hidden surfaces and edges so that they do not contribute to surface or line integrals. The difficult and time-consuming software identification of shadowed regions is 


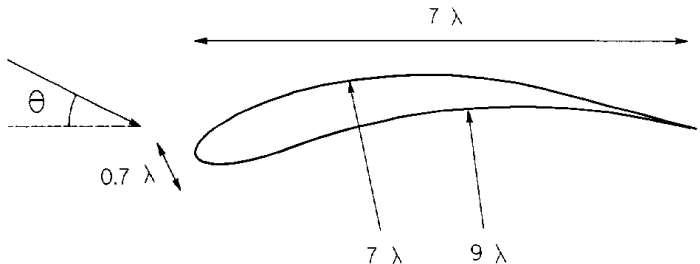

(a)

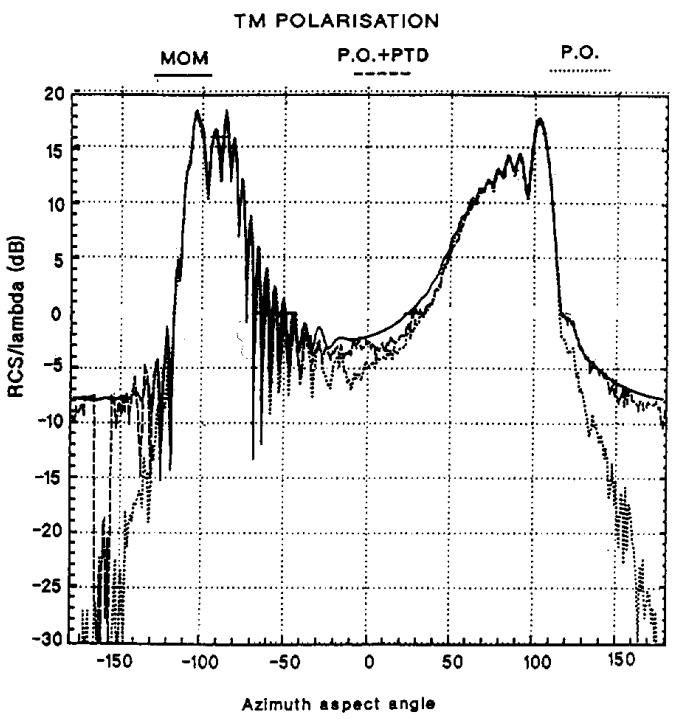

(b)

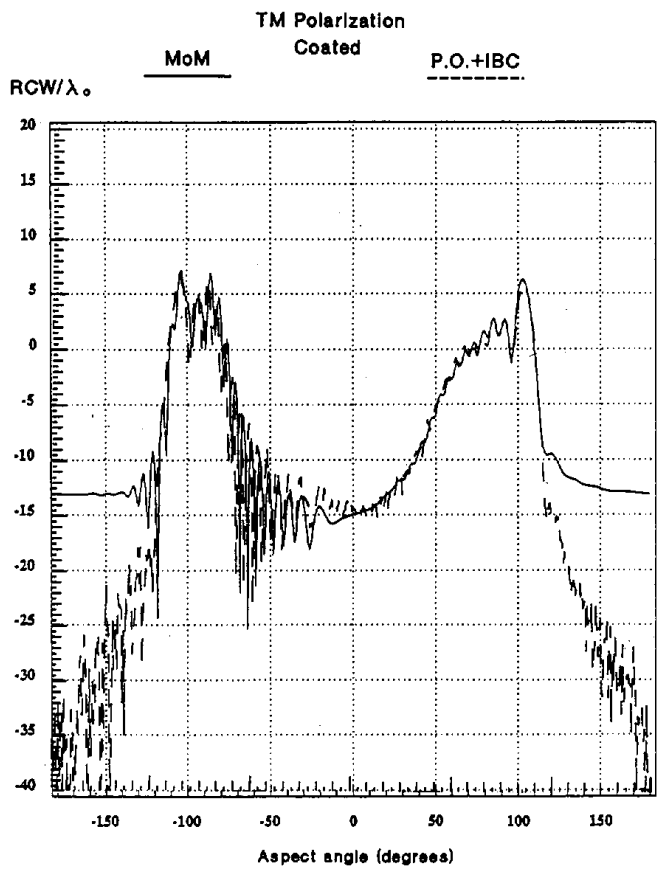

(d)
TE POLARIZATION

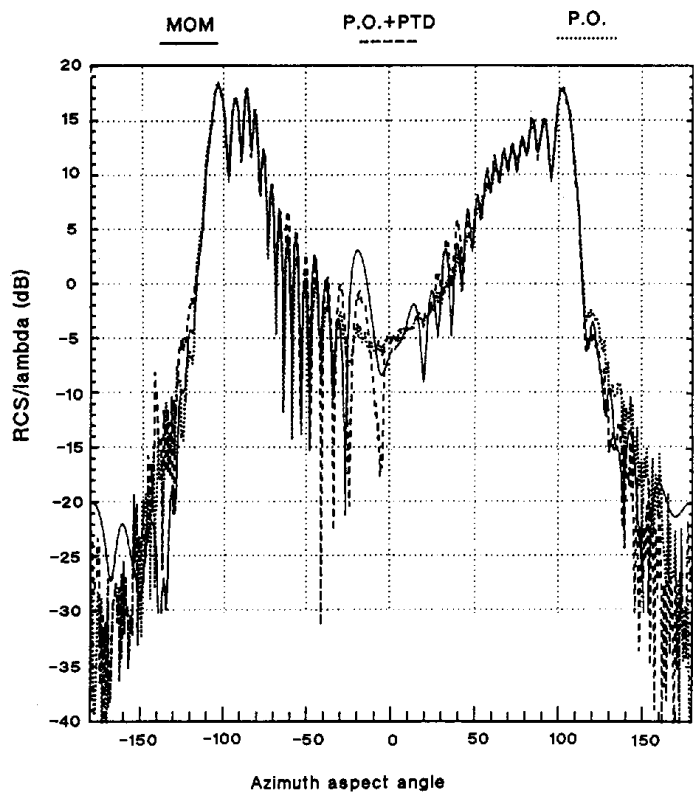

(c)

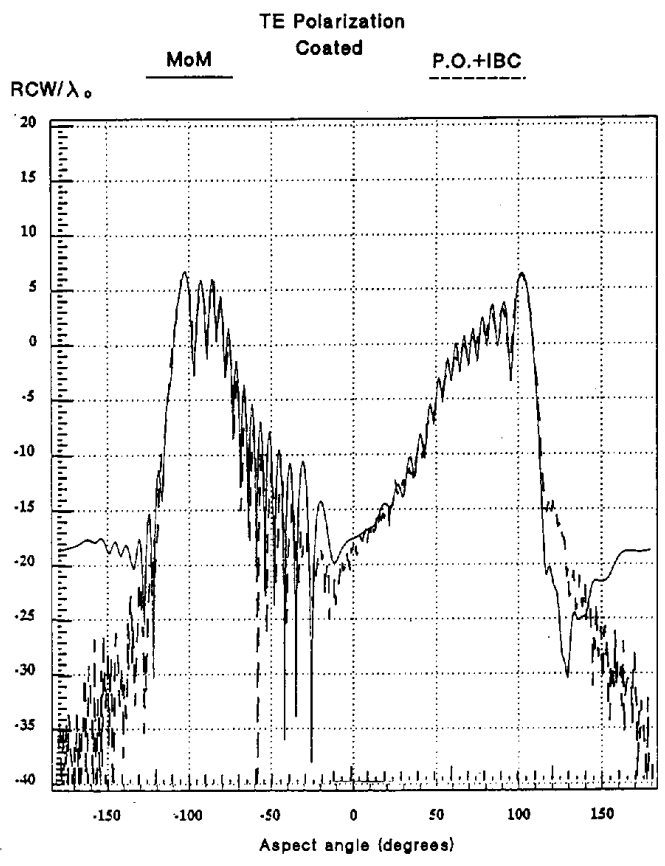

(e)

Fig. 13. (a) Two-dimensional airfoil section defined in JINA '90 workshop [23]. (b) and (c) Radar cross width (RCW) normalized by $\lambda$ for TM and TE polarizations, respectively. Results for perfectly conducting airfoil surface are compared to a rigourous solution presented by AEROSPATIALE [23]. (d) and (e) Radar cross width (RCW) normalized by $\lambda$ for TM and TE polarizations, respectively. Results for coated airfoil surface are compared to a rigourous solution presented by AEROSPATIALE [23]. The coating has a relative dielectric permitivity $\epsilon_{r}=7.4-j 1.11$, relative magnetic permeability $\mu_{r}=1.4-j 0.672$, and thickness $d=0.06 \lambda_{0}$, being $\lambda_{0}$ the wavelength in the vacuum. 
avoided, which leads to a great advantage over the classical codes for RCS prediction.

- Evaluation of surface and line integrals (PO and $\mathrm{MEC})$ by graphical processing of an image of the target at the workstation screen. As this is independent of target-complexity and electrical size, the CPU time and RAM requirements do not increase with target size or complexity.

- Potential real-time computation with a high-performance workstation and hardware-graphics accelerator, while the classical techniques require powerful super-computers in order to obtain real-time results.

- GRECO code can be integrated with CAD geometric modeling package [12], thus providing an efficient tool for interactive modeling, design, and analysis of aircraft with RCS specifications.

It must be noted that GRECO is able to analyze targets of electrical size as large as $2^{n} / 16 \lambda$, with a maximum phase error of $\lambda / 8$, where $n$ is the number of bits in which distance $z$ to the observer is discretized. This means that we can analyze $4000 \lambda$ with a usual 16-b discretization, although some workstations use 24-b $z$ discretization, or even more. The resolution in the discretization of $x, y$ 2-D screen coordinates, usually $1024 \times$ 2048 pixels, only limits the maximum complexity of the target-details must be larger than a pixel-but does not limit the target electrical size, because $x, y$ lay on a plane perpendicular to the direction of incidence and observation.

In conclusion, graphical processing is probably the optimum approach for analyzing very large and complex aircraft using high-frequency approximations. However, there are some scattering sources that at present cannot be analyzed by GRECO: cavities at engine inlets, creeping waves, discontinuities and slots over the aircraft surface, impedance wedges, etc. These effects should be analyzed by different methods and its results added to GRECO.

\section{ACKNOWLEDGMENT}

The authors would like to express their gratitude to Prof. A. Cardama for his valuable technical discussions on high-frequency scattering and to $\mathbf{M}$. Vall-llossera for her help in the development of the GRECO code.

\section{REFERENCES}

[1] E. F. Knott, J. F. Shaeffer, and M. T. Tuley, Radar Cross Section. New York: Artech House, 1985.

[2] N. N. Youssef, "Radar cross section of complex targets," Proc. IEEE, vol. 77, no. 5, May 1989.

[3] R. Abad, J. I. Casado, P. L. Primo del Val, R. Torres, M. Domingo, F. Rivas, M. F. Cátedra, "Modelos matemáticos para el estudio de la RCS debida a doble reflexión, difracción en aristas, y sombras por eclipse en parches poligonales planos," in Proc. V Symp. Nacional del Comité Español de la URSI, Vigo., Sept. 26-28, 1990, pp. 190-194.

[4] M. Domingo, F. Rivas, M. F. Cátedra, R. Abad, J. I. Casado, P. L. Primo del Val, and R. Torres, "Programa base de GTD-PTD para el cálculo de la RCS de cuerpos conductores modelados por parches planos y teniendo en cuenta simple y doble reflexión, difracción en aristas y eliminación de zonas ocultas" in Proc. $V$ Symp. Nacional del Comité Español de la URSI, Vigo., Sept. 26-28, 1990, pp. 185-189.

[5] W. B. Gordon, "Far field approximation of the Kirchhof-
Helmholtz representation of scattered fields," IEEE Trans. Antennas Propagat., vol. AP-23, no. 5, pp. 864-876, July 1975 .

[6] J. M. Rius and M. Ferrando, "Real time radar cross-section of complex targets by physical optics graphical processing," in Proc. 1990 IEEE Int. Symp. Antennas and Propagation, Dallas, May 7-11, 1990, pp. 1280-1283.

[7] J. M. Rius, M. Vall-llossera, and M. Ferrando, "Fast algorithms for radar cross section computation of complex objects," Joumées Internationales de Nice sur les Antennes JINA' 90 , Nice, France, Nov. 13-16, 1990.

[8] J. M. Rius and M. Vall-llossera, "High frequency radar cross section of complex objects in real time," in Proc. 1991 IEEE AP-S International Symposium London, Ontario, Canada, June 1991.

[9] J. M. Rius, M. Vall-llossera, and A. Cardama, "High frequency RCS of perfectly conducting or coated complex objects in real time," presented at the 21th European Microwave Conference, Stuttgart, Germany, Sept. 9-12, 1991.

[10] J. M. Rius, M. Vall-llossera, and A. Cardama, "Real time RCS of perfectly conducting or coated radar targets," presented at the $2 n d$ Int. Conf. on Electromagnetics in Aerospace Applications (ICEAA 91), Torino, Italy, Sept. 17-20, 1991.

[11] J. M. Rius, "Sección Recta de Blancos Radar Complejos en Tiempo Real," Tesis Doctoral, Universidat Politècnica de Catalunya, July 1991. Microfilm edition available from "Universitat Poltènica de Cataunya," Barcelona, Spain, 1992.

[12] “I-DEAS Geomod Users Guide," Structural Dynamics Research Corporation (SDRC), Milford, $\mathrm{OH} 45150$.

[13] C. de Boor, A Pracical Guide to Spline. New York: SpringerVerlag, 1978.

[14] G. Farin, Curves and Surfaces for Computer Aided Geometric Design: A Practical Guide. New York: Academic, 1988.

[15] M. Pizarroso, J. Pérez, and M. F. Cátedra, "Software geométrico para el ćlculo de RCS de sólidos modelados por NURBS," in Proc. $V$ Symp. Nacional del Comité Español de la URSI, Vigo, Sept. 26-28, 1990, pp. 195-199.

[16] X. Fernández Hermida and A. García Pino, "Spline modeling to compute de RCS of arbitrarily shaped cavities by the GO/AI method," in Proc. IEEE / URSI Meeting, Dallas, May 7-11, 1990, p. 280 .

[17] J. M. Alvarez, J. C. Cruellas, and M. Ferrando, "A hibrid modalboundary element method for electromagnetic scattering from arbitrary conducting wedges," in Proc. 1990 IEEE Int. Symp. Antennas and Propagation, Dallas, May 7-11, 1990, pp. 1288-1291.

[18] B. R. Dewey, Computer Graphics for Engineers. New York: Harper \& Row, 1988.

[19] B. T. Phong, Illumination for computer generated images, Ph.D. dissertation, Univ. Utah, 1973.

[20] A. L. Maffet, Topics for a Statistical Description of Radar Cross Section. New York: Wiley, 1989.

[21] D. Klement, J. Peissner, and V. Stein, "Special problems in applying the physical optics method for backscatter computations of complicated objects," IEEE Trans. Antennas Propagat., vol. AP-36, no. 2, pp. $228-237$, Feb. 1988.

[22] R. A. Shore and A. D. Yaghjian, "Incremental diffraction coefficients for planar surfaces," IEEE Trans. Antennas Propagat., vol. AP-36, no. 1, Jan. 1988.

[23] Workshop on RCS of perfectly conducting or coated bodies, Dassault Aviation, Société Mothesim and CNET-PAB La Turbie, Nice, France, Nov. 16, 1990.

[24] E. N. Jacobs, K. E. Ward, and R. M. Pinkerton, "The characteristics of 78 related airfoil sections from tests in the variabledensity wind tunnel," Nat. Advisory Comm. Aeronautics, Washington, DC, NACA Tech. Rep. 460, 1933.

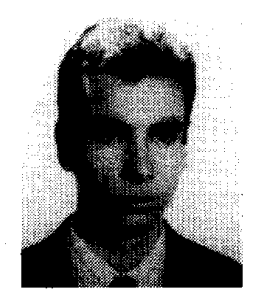

Juan M. Rius (S'89-M'92) was born in Barcelona, Spain, in 1963. He received the Ingeniero degree in telecommunication engineering in 1987 and the Doctor Ingeniero degree in 1991, both from the Universitat Politècnica de Catalunya, Barcelona, Spain.

In 1985 he joined the Antennas, Microwave and Radar Group (AMR) at the UPC as an Undergraduate Research Assistant. From 1985-1987 he worked on the development of cylindrical geometry algorithms for microwave tomography. Currently he is Associate Professor at the UPC. Since 1988 
he has been engaged in the development of the graphical processing technique for high-frequency RCS prediction. His current research interests involve both high frequency techniques and numerical methods for electromagnetic scattering analysis.

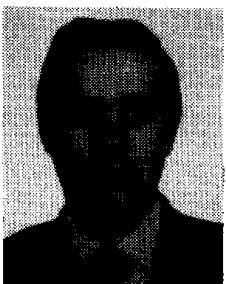

Miguel Ferrando (S'81-M'83) received the "Ingeniero de Telecommunicación" degree and the "Doctor" degree from the Barcelona Polytechnic University, Spain, in 1977 and 1982, respectively.

In 1990 he joined the Polytechnic University, Spain, where he is currently Dean of the Telecommunication Engineering School and Professor of Antennas and Satelite communications. He has been envolved in research activiantennas, and satellite systems.

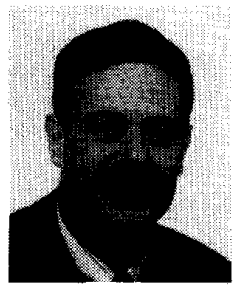

Luis Jofre (S'79-M'83) was born in Mataró, Spain, in 1956. He received the Ingeniero and Doctor Ingeniero degrees in telecommunication engineering, both from the Polytechnic University of Catalonia (UPC), Barcelona, Spain, in 1978 and 1982 , respectively.

In 1978 he was Research Assistant in the Electrophysics group at UPC, where he worked on the analysis and the near-field measurement of antennas. In 1981 he joined the Ecole Supérieure d'Electricité in Paris, where he was involved in microwave imaging techniques for biomedical applications. During the period 1986-1987 he was a Visiting Fulbright Scholar at the Georgia Institute of Technology, Atlanta, working on antenna measurement and electromagnetic imaging. He is currently Professor and Director of the Telecommunication Engineering School at UPC where he is engaged in research on antennas and electromagnetic scattering and imaging, both numerical and experimental aspects. 